\title{
Deception and Self-Awareness
}

\author{
Glyn Lawson ${ }^{1}$, Alex Stedmon ${ }^{1}$, Chloe Zhang ${ }^{2}$, Dawn Eubanks ${ }^{2}$ and Lara Frumkin \\ ${ }^{1}$ Human Factors Research Group, Faculty of Engineering, The University of Nottingham, \\ United Kingdom, NG7 2RD \\ ${ }^{2}$ School of Management, University of Bath, United Kingdom, BA2 7AY \\ ${ }^{3}$ School of Psychology, The University of East London, United Kindgom, E15 4LZ \\ \{glyn.lawson, alex.stedmon\}@nottingham.ac.uk, \{kz222,d.eubanks\}@bath.ac.uk, \\ 1.frumkin@uel.ac.uk
}

\begin{abstract}
This paper presents a study conducted for the Shades of Grey EPSRC research project $(\mathrm{EP} / \mathrm{H} 02302 \mathrm{X} / 1)$, which aims to develop a suite of interventions for identifying terrorist activities. The study investigated the body movements demonstrated by participants while waiting to be interviewed, in one of two conditions: preparing to lie or preparing to tell the truth. The effect of self-awareness was also investigated, with half of the participants sitting in front of a full length mirror during the waiting period. The other half faced a blank wall. A significant interaction was found for the duration of hand/arm movements between the deception and self-awareness conditions $(F=4.335$, $\mathrm{df}=1 ; 76, \mathrm{p}<0.05)$. Without a mirror, participants expecting to lie spent less time moving their hands than those expecting to tell the truth; the opposite was seen in the presence of a mirror. This finding indicates a new research area worth further investigation.
\end{abstract}

Keywords: terrorism, deception, self-awareness

\section{$1 \quad$ Introduction}

Recent statistics have shown that arrests associated with terrorism are rising, with 1,759 arrests occurring since September 11, 2001 [1]. In particular, intent to commit a terrorist act has increased by $30 \%$ since 2001 . Terrorist attacks involving large-scale, high-value targets and widespread influences are considered strategic attacks, which involve a planning phase, including the processes of intelligence, surveillance and reconnaissance (ISR) [2, 3]. Most of the terrorist attack-planning indicators are hard to detect [3], and terrorists tend to behave differently based on their environments. However, at certain stages in ISR terrorists may be physically-present at their intended target [3] and need to conceal their intentions. This provides opportunities to identify suspicious individuals during the pre-attack stage using detection approaches.

The Shades of Grey research project aims to develop scientific interventions which will work on eliciting robust, reliable and operational indicators of suspicious behaviors, particularly relating to the reconnaissance stage of terrorist activities. This paper is associated with a work package which will develop and assess the value of different types of interventions, specifically aimed at revealing deception-related 
factors falling into the broad category of non-verbal behaviors. These behavioral cues might be aroused by intervention strategies designed to amplify suspicious reactions, in particular during reconnaissance of a terrorist attack in public areas.

Considering previous work on cues to deception, the Multi-Factor Model [4] proposes three factors which the influence behavioral cues to deception: emotion, cognitive effort, and attempted behavioral control. These factors also feature different aspects of deception, and the strength of such factors is highly relevant to cues associated with lying. These will be described below.

Ekman [5] argued that there are three different types of emotion associated with deception: fear, guilt and duping delight. Each factor that elicits emotional cues can occur all at once or in succession. Fear and excitement (the latter occurring through duping delight) might result in signs of arousal, such as increases in limb movements, speech fillers, and speech errors [6]. Guilt might result in gaze aversion [6]. Excitement may also result in signs of joy, such as smiling [7]. In spite of these deception cues, it is believed that liars try to use other facial expressions to mask signs of the emotion that they intended to conceal, in which case the effort of masking might fail [8]. Thus emotional leakage — which can be shown by facial expression [5, 9] or body movement - is a crucial non-verbal cue to deception.

Lying sometimes requires extra mental and cognitive effort than truth-telling. Because deceivers might be pre-occupied by formulating lies as well as remembering to play their role, they need to pay special attention to their behavior as well as monitoring the reaction of their targets, and they have to suppress the truth when they are lying. These processes for lying all require cognitive demand [6]. Deliberate efforts to "fight with" the conflict between lies and truth in their minds place mental demands upon liars e.g., $[10,11]$. Evidence provided by neuroimaging studies (e.g., [12]) supports this point of view: the prefrontal cortex and anterior cingulate cortex are related to deception, which are involved in processing complex cognitive tasks and cognitive conflict. In addition, Carrión, Keenan, and Sebanz [13] revealed that tracing the target's mental state leads to greater cognitive demands compared with the conflict of the content of true or false statements.

People engaged in cognitive complexity present fewer hand and arm movements [8], less blinking [14], more [8] or less gaze aversion [15], and more speech hesitation [16] and errors [17]. They might display more pauses in speech, speak with a lower voice, and have longer reaction times, all of which are also found to be related to cognitive load [6]. The concentration which is aroused by cognitive overload thus influences behavior, such as the decrease in body movements, since the high cognitive demand leads to the neglect of body language [6]. As a consequence of cognitive overload, liars might be more rigid during deception (e.g., [17]; this is also caused by physiological inhibition from certain brain areas [18].

Concerning behavioral control, liars adjust their behaviors during lying by monitoring the reactions from their targets [19]. It is proposed that perceiving, monitoring and communicating with targets helps liars to successfully deceive (e.g., $[20,21]$. Notably, in order to appear honest or normal, liars may attempt to control their behaviors during deception. Some evidence shows that liars may try to exhibit behaviors which they believe are credible, such as trying to behave positively and friendly to convince their targets [17]. However, such kind of deliberate selfregulation sometimes makes liars look over-controlled [6]. Some reviews [7, 17] 
indicate that liars' behavior might look rigid and tense, but speech might sound too smooth (presents less disturbances due to over control of speech) [16]. Furthermore, they might also be less forthcoming and less pleasant [17]. The complex presence of attempted behavioral control varies by person to person and it could be influenced by the simultaneous effect of emotion and cognitive load.

As described above, previous work has often been based on participants' behavior during interviews in which they are required to act deceptively. This study aimed to investigate cues to deception exhibited by people as they prepare to act deceptively in an interview. The outcome of this research could be used to support security personnel as they observe suspects prior to interview. Of relevance to behavioral control, this study also attempts to investigate the influence of self-awareness on deception cues.

\section{$2 \quad$ Method}

\subsection{Participants}

Recruitment was conducted by participant self-selection in response to posters and emails. Adverts specified that only undergraduate students should apply, and that they should not suffer from any mental ill-health. This requirement was to minimize the impact of any potential distress experienced from expecting to lie in the deception condition. 80 participants were recruited, 39 female and 41 male (mean age $=20$, $\mathrm{SD}=1.30$, range $=18-24)$.

\subsection{Apparatus/equipment}

The experiment was conducted in a small office area. This contained a reception area and an interview area with chairs for the participant and the interviewer. All objects were removed from the walls to create an environment which was relatively free from distractions. There were no windows looking into or out of the interview area.

A camcorder was hidden within a green box file adjacent to the interview area. The aperture in the file had to be widened to enable the camera to capture then entire body of the participants. Because of this, it was possible to identify the lens, but only with close attention. Typical office products (glue stick, CD, marker pen) were located around the aperture to divert the participants' attention from the lens.

\subsection{Experimental Design}

The experiment took the form of a $2 * 2$ between-subjects design. The two independent variables were:

- Self-awareness: mirror/no mirror. In the mirror condition, a full-length mirror was located directly opposite the participants in the waiting area. The mirror was removed and hidden for the no mirror condition. 
- Deception: truth/lying. In the truth condition, participants were told to answer all questions truthfully. In the lying condition they were told that they could not answer any of the questions asked by the interviewer truthfully.

Thus, there were a total of four experimental conditions, of which participants were randomly assigned to one:

1. Mirror and truth (participants expect to answer truthfully)

2. Mirror and lying (participants have to invent answers and expect to answer untruthfully)

3. No mirror and truth

4. No mirror and lying

\subsection{Procedure}

Participants were invited to take part in a trial to investigate deception skills in interview. Prior to each session, the hidden video camera was started. Upon arrival, participants were asked to sit in the reception area. A researcher explained to each participant that the study was being conducted to investigate deception skills in interview, and that after completing some preliminary forms and questionnaires an interviewer would arrive and ask questions about their degree courses. They were told that they should either answer truthfully (truth condition) or lie in all their answers (lying condition). Participants were asked to sign a consent form agreeing that they were willing to continue.

The researcher then led the participant to interview area. The researcher told the participant that they were leaving to find the interviewer and that they would return at the end of the session to complete participant payment forms. The researcher left the room under the pretence of going to find the interviewer. In reality, they hid outside the laboratory and timed five minutes. After this period, the researcher re-entered the room, apologized for the delay and asked the participant to return to the reception area. They told the participant that in fact there was not going to be an interview. After completing payment forms, and explaining the true purpose of the study, the hidden camera was stopped.

\section{$3 \quad$ Results}

The video footage of the 80 participants was coded using the Observer software. One researcher coded all footage. The coding scheme used is shown in Table 1. This was based on previous research into cues to deception, but was simplified due to the practical requirements for coding. Note that hand or arm movement includes any finger, hand or arm movement on either left, right or both sides; similarly foot or leg includes movement on left, right or both sides. 
Table 1. Coding scheme

\begin{tabular}{cccc}
\hline $\begin{array}{c}\text { Hand or arm (either left } \\
\text { or right) }\end{array}$ & $\begin{array}{c}\text { Foot or leg (either left } \\
\text { or right) }\end{array}$ & $\begin{array}{c}\text { Whole body or } \\
\text { torso }\end{array}$ & Gaze direction \\
\hline Moving & Moving & Moving & Directly forwards \\
Still & Still & Still & $\begin{array}{c}\text { Towards camera } \\
\text { Other }\end{array}$ \\
\hline
\end{tabular}

8 participants $(10 \%)$ were randomly selected for coding by a second researcher to investigate inter-rater reliability. These were not used in the analysis of the behaviors, only to investigate the reliability. Cohen's Kappa, as calculated using the Observer software, was found to be significant, and towards the upper limits of "moderate" agreement $(\mathrm{Kappa}=0.57 ; \mathrm{p}<0.01)$. As the main results of interest included durations and frequencies these were also investigated. The durations were summed for the movement categories for the eight participants. This was repeated for the second rater; the durations were found to be highly correlated between the raters $\left(r_{p}=0.965, N=6\right.$, $\mathrm{p}<0.01)$. This process was repeated for the frequencies of the behaviors, which was also found to be highly correlated $\left(r_{p}=0.923, N=6, p<0.01\right)$. Thus, the results were deemed sufficiently reliable for further analysis.

The results are shown below, structured according to the movement categories in Table 1. Within each section the analyses are shown for the duration (i.e. total time spent moving) and frequency (i.e. total number of times the body part was moved regardless of duration) of movements.

\subsection{Hand/arm movements}

Hand and arm movement was first investigated using a $2 * 2$ between-subjects ANOVA. The ANOVA for duration of the movements is shown in Table 2. This demonstrates a significant interaction between deception and self-awareness. The interaction plot is shown in Figure 1. This shows that without a mirror, participants expecting to tell the truth spend more time moving their hands than those expecting to lie; the opposite is seen in the presence of a mirror.

Table 2. ANOVA for duration of hand/arm movements

\begin{tabular}{ccccc}
\hline Effect & $\mathrm{F}$ & $\mathrm{df}$ & $\mathrm{p}$ & $\mathrm{Eta}^{2}$ \\
\hline Deception level & 0.034 & 1,76 & $\mathrm{NS}$ & 0.000 \\
Self-awareness & 0.280 & 1,76 & $\mathrm{NS}$ & 0.004 \\
Deception*self-awareness & 4.335 & 1,76 & $<0.05$ & 0.054 \\
\hline
\end{tabular}




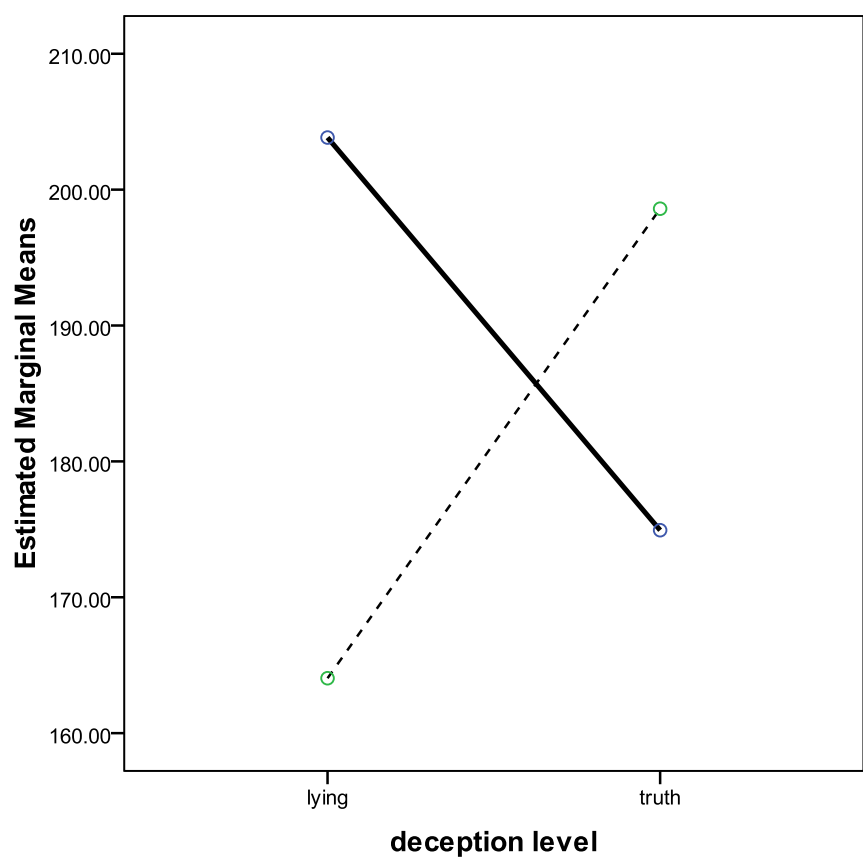

self awareness

- mirror

$-\cdots$ no-mirror

Fig. 1. Interaction plot for duration of hand/arm movements: deception*self-awareness

The results of the $2 * 2$ ANOVA for frequency of hand/arm movements is shown in Table 3. There were no significant main effects or interaction.

Table 3. ANOVA for frequency of hand/arm movements

\begin{tabular}{ccccc}
\hline Effect & $\mathrm{F}$ & $\mathrm{df}$ & $\mathrm{p}$ & $\mathrm{Eta}^{2}$ \\
\hline Deception level & 1.045 & 1,76 & $\mathrm{NS}$ & 0.014 \\
Self-awareness & 3.473 & 1,76 & $\mathrm{NS}$ & 0.044 \\
Deception*self-awareness & 1.305 & 1,76 & $\mathrm{NS}$ & 0.017 \\
\hline
\end{tabular}

\subsection{Leg/foot movements}

No significant main effects or interactions were found for duration or frequency of leg/foot movements (Tables 4 and 5).

Table 4. ANOVA for duration of leg/foot movements

\begin{tabular}{ccccc}
\hline Effect & $\mathrm{F}$ & $\mathrm{df}$ & $\mathrm{p}$ & $\mathrm{Eta}^{2}$ \\
\hline Deception level & 0.386 & 1,76 & $\mathrm{NS}$ & 0.005 \\
Self-awareness & 0.807 & 1,76 & $\mathrm{NS}$ & 0.011 \\
Deception*self-awareness & 2.125 & 1,76 & $\mathrm{NS}$ & 0.027 \\
\hline
\end{tabular}


Table 5. ANOVA for frequency of leg/foot movements

\begin{tabular}{ccccc}
\hline Effect & $\mathrm{F}$ & $\mathrm{df}$ & $\mathrm{p}$ & Eta $^{2}$ \\
\hline Deception level & 0.767 & 1,76 & $\mathrm{NS}$ & 0.010 \\
Self-awareness & 0.971 & 1,76 & NS & 0.013 \\
Deception*self-awareness & 2.698 & 1,76 & NS & 0.034 \\
\hline
\end{tabular}

\subsection{Whole body/torso movements}

A main effect of self-awareness was found for duration of whole body/torso movements (Table 6). Those with the mirror spent longer moving (mean duration: $28.584 \mathrm{~s} ; \mathrm{SD}=46.192$ ) than those without the mirror (mean duration: $12.225 \mathrm{~s}$; $\mathrm{SD}=11.838$ ). There were no significant findings for the frequency of whole body/torso movements (Table 7).

Table 6. ANOVA for duration of whole body/torso movements

\begin{tabular}{ccccc}
\hline Effect & F & df & p & Eta $^{2}$ \\
\hline Deception level & 3.635 & 1,76 & NS & 0.046 \\
Self-awareness & 5.035 & 1,76 & $\mathrm{p}<0.05$ & 0.062 \\
Interaction deception*self-awareness & 3.789 & 1,76 & $\mathrm{NS}$ & 0.047 \\
\hline
\end{tabular}

Table 7. ANOVA for frequency of whole body/torso movements

\begin{tabular}{ccccc}
\hline Effect & F & df & p & Eta $^{2}$ \\
\hline Deception level & 0.137 & 1,76 & NS & 0.002 \\
Self-awareness & 3.423 & 1,76 & NS & 0.043 \\
Interaction deception*self-awareness & 1.232 & 1,76 & NS & 0.016 \\
\hline
\end{tabular}

\subsection{Gaze direction}

As gaze direction was a more complex measure than the previous behaviors, this was investigated using a $2 * 2 * 3$ mixed ANOVA, with the variables of deception (expecting to lie/expecting to tell the truth), self-awareness (mirror/no mirror) and gaze direction (directly forwards/towards camera/other).

Table 8. ANOVA for duration of gaze direction

\begin{tabular}{ccccc}
\hline Effect & $\mathrm{F}^{\mathrm{a}}$ & $\mathrm{df}$ & $\mathrm{p}$ & Eta $^{2}$ \\
\hline Gaze direction & 422.469 & 2,75 & $\mathrm{p}<0.001$ & 0.918 \\
Direction*deception & 1.577 & 2,75 & $\mathrm{NS}$ & 0.040 \\
Direction*self-awareness & 24.578 & 2,75 & $\mathrm{p}<0.001$ & 0.396 \\
Direction*deception*self-awareness & 0.251 & 2,75 & $\mathrm{NS}$ & 0.007 \\
deception & 0.616 & 1,76 & $\mathrm{NS}$ & 0.008 \\
Self-awareness & 4.698 & 1,76 & $\mathrm{p}<0.05$ & 0.058 \\
Deception*self-awareness & 0.614 & 1,76 & $\mathrm{NS}$ & 0.008 \\
\hline
\end{tabular}

a Pillai's Trace 
For durations, a main effect was seen for gaze direction, with most time spent looking at "other" (mean=205.681; $\mathrm{SD}=67.350)$ followed by "forward" (mean=66.914; $\mathrm{SD}=67.001$ ) and finally looking towards the "camera" (mean=28.440; $\mathrm{SD}=23.957$ ). The main effect for self-awareness was simply a result of measurement tolerances, and provides no meaningful data for understanding deception behavior.

A significant interaction for gaze direction and self-awareness can also be seen in Table 8. This finding indicates a change in gaze direction in the presence/absence of a mirror.

For frequencies, the main effect of gaze direction was found to be significant with the highest frequency for "other" (mean=11.92; $\mathrm{SD}=5.233$ ) followed by "camera" (mean=7.37; $\mathrm{SD}=3.921$ ) and finally "forward" (mean=6.79; $\mathrm{SD}=5.125$ ). The interaction between direction and self-awareness was found to be significant, which also indicates a change in gaze direction in the presence/absence of a mirror.

Table 9. ANOVA for frequency of gaze direction

\begin{tabular}{ccccc}
\hline Effect & $\mathrm{F}^{\mathrm{b}}$ & $\mathrm{df}$ & $\mathrm{p}$ & $\mathrm{Eta}^{2}$ \\
\hline Gaze direction & 114.462 & 2,75 & $\mathrm{p}<0.001$ & 0.753 \\
Direction*deception & 0.228 & 2,75 & $\mathrm{NS}$ & 0.006 \\
Direction*self-awareness & 12.595 & 2,75 & $\mathrm{p}<0.001$ & 0.251 \\
Direction*deception*self- & 1.839 & 2,75 & $\mathrm{NS}$ & 0.047 \\
awareness & & & & \\
Deception level & 0.580 & 1,76 & $\mathrm{NS}$ & 0.008 \\
Self-awareness & 6.707 & 1,76 & $\mathrm{p}<0.05$ & 0.081 \\
Interaction deception*self- & 1.412 & 1,76 & $\mathrm{NS}$ & 0.018 \\
$\quad$ awareness & & & & \\
\hline
\end{tabular}

\section{Discussion}

This study indicated that few differences were observed in body movements between participants expecting to act deceptively and those expecting to tell the truth. The most notable finding was an interaction between self-awareness and deception for the duration of hand/arm movements: those in the lying condition moved their arms more in the presence of a mirror; the opposite was true for the truth tellers. It is difficult to understand why this interaction occurred, although it is certainly interesting that the presence of the mirror appears to magnify duration of the hand-arm movements of those expecting to lie. Previous research has demonstrated that people engaged in cognitive complexity (associated with lying) present fewer hand and arm movements [8].

The mirror resulted in an increase in whole body/torso movements. This may not be useful for identifying those expecting to lie, but contributes to an understanding of how people behave with increased levels of self-awareness. Similarly, gaze direction, and the interaction between gaze direction and self-awareness were significant, but these findings do not provide information with obvious use for detecting terrorist behavior.

${ }^{b}$ Pillai's Trace 
Despite the finding that none of the body movements showed a main effect of deception level (truth telling vs. lying), the interaction in hand/arm movements suggests that the notion of self-awareness is worth further investigation as a possible tool for detecting deception. Future work could investigate in further detail the specific hand/arm movements in each condition (e.g. fold arms, tap fingers, touch face), to determine whether a certain type was more prevalent in each. For practicality this study used a high-level behavioral coding scheme, which could be broken down into further sub-categories for more detailed analysis. Behaviors could also be coded in a more subjective approach, for example focusing on behaviors associated with categories such as vanity, practice, nervousness etc.

Perhaps one further aspect to consider in future work is higher stakes. The only stakes in this experiment were participants' desire to convince the experimenter that they were telling the truth. With greater stakes the results may have been different [17].

\section{Conclusions}

This paper was an initial investigation into behaviors associated with deception while participants waited to be interviewed; previous research has generally focused on the behaviors demonstrated during an interview. This study also investigated the effects of self-awareness on cues to deception. An interaction was identified between deception and self-awareness for the duration of hand/arm movements $(F=4.335$, $\mathrm{df}=1 ; 76, \mathrm{p}<0.05)$. Liars moved their hands for longer when a mirror was present. This finding suggests that further research is required to understand the effects of selfawareness on non-verbal behaviors associated with deception, and in particular prior to the deceptive event itself. This research may ultimately improve the capability of security personnel to detect terrorists or people acting deceptively.

\section{References}

1. Home office statistical bulletin. Retrieved from www.statistics.gov.uk.

2. Jessee, D. Tactical means, strategic ends: al qaeda's use of denial and deception. Terrorism and Political Violence. 18, 367-388 (2006)

3. O'Brien, K. A. Assessing hostile reconnaissance and terrorist intelligence activities. The RUSI Journal, 153, 34-39 (2008)

4. Zuckerman, M., DePaulo, B. M., \& Rosenthal, R. Verbal and nonverbal communication of deception. In L. Berkowitz (Ed.), Advances in Experimental Social Psychology (Vol. 14, pp. 1--57). Academic Press, New York (1981)

5. Ekman, P.. Telling lies: Clues to deceit in the marketplace, politics, and marriage (1st ed.), pp. 43--161. Norton, New York (1985)

6. Vrij, A. Detecting lies and deceit: pitfalls and opportunities (2nd ed.). pp. 1--188. Wiley, West Sussex (2008).

7. Memon, A., Vrij, A., \& Bull, R. Psychology and law: truthfulness, accuracy and credibility (2nd ed.). pp. 1--55. Wiley, Chichester (2003) 
8. Ekman, P. Deception, lying and demeanor. In: D. F. Halpern \& A. Voiskunskii (Eds.), States of mind: American and post-soviet perspectives on contemporary issues in psychology, pp. 93--105. Oxford University Press, Oxford (1997)

9. Ekman, P., \& O'Sullivan, M. From flawed self-assessment to blatant whoppers: the utility of voluntary and involuntary behavior in detecting deception. Behavioral Sciences \& the Law, 24, 673-686 (2006)

10. Walczyk, J. J., Roper, K. S., Seemann, E., \& Humphrey, A. M. Cognitive mechanisms underlying lying to questions: Response time as a cue to deception. Applied Cognitive Psychology, 17, 755-774 (2003)

11. Walczyk, J. J., Schwartz, J. P., Clifton, R., Adams, B., Wei, M., \& Zha, P. Lying person-to-person about life events: A cognitive framework for lie detection. Personnel Psychology, 59, 141-170 (2005)

12. Kozel, F., Johnson, K., Mu, Q., Grenesko, E., Laken, S., \& George, M. Detecting deception using functional magnetic resonance imaging. Biological Psychiatry, 58, 605--613 (2005)

13. Carrión, R. E., Keenan, J. P., \& Sebanz, N. A truth that's told with bad intent: an ERP study of deception. Cognition, 114, 105--110 (2010).

14. Bagley, J., \& Manelis, L. Effect of awareness on an indicator of cognitive load. Perceptual and Motor Skills, 49, 591-594 (1979)

15. Doherty-Sneddon, G., \& Phelps, F. G. Gaze aversion: A response to cognitive or social difficulty? Memory \& Cognition, 33, 727-733 (2005)

16. Vrij, A., \& Heaven, S. Vocal and verbal indicators of deception as a function of lie complexity. Psychology, Crime \& Law, 5, 203-215 (1999)

17. DePaulo, B. M., Lindsay, J. J., Malone, B. E., Muhlenbruck, L., Charlton, K., \& Cooper, H. Cues to deception. Psychological Bulletin, 129, 74--118 (2003)

18. Vrij, A., Fisher, R., Mann, S., \& Leal, S. Detecting deception by manipulating cognitive load. Trends in Cognitive Sciences, 10, 141--142 (2006).

19. Buller, D., \& Burgoon, J. Interpersonal deception theory. Communication Theory, 6 , 203--242 (1996)

20. Burgoon, J., Blair, J., \& Strom, R. Cognitive biases and nonverbal cue availability in detecting deception. Human Communication Research, 34, 572--599 (2008).

21. Burgoon, J., Buller, D., \& Floyd, K. Does participation affect deception success? Human Communication Research, 27, 503-534 (2001).

\section{Acknowledgements.}

The authors acknowledge the EPSRC for funding this work. 